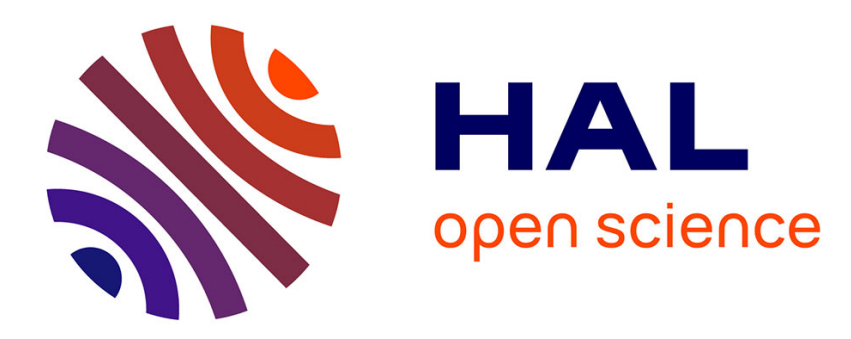

\title{
Le portrait d'auteur dans les manuscrits hippiatriques byzantins
}

\author{
Stavros Lazaris
}

\section{To cite this version:}

Stavros Lazaris. Le portrait d'auteur dans les manuscrits hippiatriques byzantins. KTÈMA Civilisations de l'Orient, de la Grèce et de Rome antiques, 2009, 34, pp.307-318. halshs-00462088

\section{HAL Id: halshs-00462088 \\ https://shs.hal.science/halshs-00462088}

Submitted on 23 Mar 2010

HAL is a multi-disciplinary open access archive for the deposit and dissemination of scientific research documents, whether they are published or not. The documents may come from teaching and research institutions in France or abroad, or from public or private research centers.
L'archive ouverte pluridisciplinaire HAL, est destinée au dépôt et à la diffusion de documents scientifiques de niveau recherche, publiés ou non, émanant des établissements d'enseignement et de recherche français ou étrangers, des laboratoires publics ou privés. 


\section{Le portrait d'auteur dans les manuscrits hippiatriques byzantins}

RÉsumé.- Parmi les portraits que l'on rencontre dans l'art médiéval byzantin, l'un des plus répandus est celui de l'auteur. Relativement stéréotypé, il se confond le plus souvent avec celui de l'auteur-scribe représenté au travail. On le voit en train d'écrire sur un rouleau ou un codex ou encore de copier d'un support sur l'autre, de dicter son cuvre à un copiste, de la présenter au lecteur. Or le portrait qui se trouve dans les deux seuls manuscrits grecs hippiatriques illustrés présente une tout autre iconographie, qui le différencie du portrait de l'auteur-scribe. Bien que les deux miniaturistes aient suivi des voies différentes, ils ont voulu l'un et l'autre mettre en avant l'autorité de l'auteur et l'excellence de son ceuvre. L'examen des attributs et de la gestuelle adoptés par chaque imagier permettra de comprendre les raisons de leur choix: celui du portrait de l'auteursavant.

ABSTRACT.- Amongst the different portraits found in Medieval Byzantine Art, the author's portrait is one of the most common. Relatively stereotyped, it is often mistaken for the portrait of the author-scribe represented working. We can see him writing on a roll or on a codex or even copying from one to the other, dictating his work to a copyist, or presenting it to the reader. Now, the portrait the only two Greek hippiatric manuscripts which are illustrated has a very different iconography than the one of the author-scribe. Despite the fact that the two miniaturists have followed different ways, they both wanted to highlight the authority of the author and the excellence of his work. The analysis of the attributes and the gestures used by each of the miniaturists will allow us to understand the reasons behind their choice: the use of an author-scholar's portrait.

Deux traités hippiatriques grecs illustrés nous sont parvenus dans deux manuscrits byzantins tardifs ${ }^{1}$ : le Leidensis Vossianus graecus $Q .50$ (ff. $5^{\mathrm{r}-90^{\mathrm{r}}}$ et $90^{\mathrm{r}}-144^{\mathrm{v}}$ ) du milieu du XIV ${ }^{\mathrm{e}}$ siècle ${ }^{2}$ et le Parisinus graecus 2244 (ff. $1^{\mathrm{r}-62^{\mathrm{v}}}$ et $62^{\mathrm{v}}-74^{\mathrm{v}}$ ) de la fin de ce même siècle ${ }^{3}$. Le premier écrit est une tentative maladroite de recomposition de l'œuvre originale de Hiéroclès, fonctionnaire byzantin

(1) Plusieurs chercheurs utilisent, pour désigner les ornements contenus dans un autre manuscrit grec hippiatrique (Berolinensis Phillippicus 1538), le terme «illustration ». Or, ce manuscrit ne contient que des en-têtes de chapitres purement ornementaux, en forme de portiques, décorés de motifs végétaux ou géométriques. K. WeitzmanN, Geistige Grundlagen und Wesen der Makedonischen Renaissance [Arbeitsgemeinschaft für Forschung des Landes Nordrhein-Westfalen. Geisteswissenschaften, 107], Cologne, 1963, p. 24, a essayé de démontrer qu'à l'origine ce manuscrit était pourvu d'un livret rempli d'illustrations mais, à mon avis, sans preuve décisive. Je pense que des manuscrits comme l'Evangile de Rossano, où toutes les miniatures ont été rassemblées en tête du codex, sont exceptionnels, du moins dans l'aire byzantine.

(2) Sur ce manuscrit, voir K. A.DEMEYIER, Codices Vossiani Graeci et Miscellamei [Bibliotheca Universitatis Leidensis. Codices Manuscripti, 6], Leyde, 1955, p. 158-159. L'auteur propose le milieu du XIVe siècle d'après l'étude des filigranes, mais également le XVe siècle, au début du catalogue, sans précision supplémentaire. A. W. Brvanck, Les principaux manuscrits a peintures conservés dans les collections publiques du Royaume des Pays-Bas [Bulletin de la Société française de reproductions de manuscrits à peintures], Paris, 1931, p. 78, date le manuscrit, sans autre explication, des alentours de 1470 .

(3) Sur ce manuscrit, voir S. LAzarIS, "Le Parisinus graecus 2244, ff. 1-74. Un exemple d'illustration scientifique à Byzance ${ }^{2}$ Etudes balkaniques. Cahiers Pierre Belon, 2 (1995), notamment p. 163-167 (http://halshs.archives-ouvertes.fr/ docs/00/04/26/84/PDF/SL.Illustrationcomplet.pdf). 
qui vécut vers la fin du IVe siècle 4 . Il s'est appuyé en très grande partie sur l'œuvre d'Apsyrtos, vétérinaire militaire du début de ce même siècle ${ }^{5}$, pour rédiger son texte. Le second traité est une sorte d'abrégé de la Collection hippiatrique grecque, connu, depuis les travaux de G. Björck, sous le nom d'Épitomét. En accord avec la thématique générale de la journée d'étude dont ce volume publie les Actes, le présent travail portera sur un point bien précis de l'illustration: le "portrait» d'un personnage qui se trouve dans l'œuvre recomposée de Hiéroclès ${ }^{7}$.

Le portrait est un genre bien défini. Comme le souligne L. Braun «Le portrait n'est pas un double, et ne veut l'être [...]. Le portrait est toujours une transposition: à partir du modèle le peintre ou le dessinateur ou le photographe crée un simulacre; et de ce simulacre nous disons qu'il re-présente le sujet: il le présente à nouveau, mais autrement. C'est dans cet adverbe que réside la problématique [...] de la mimesis. ${ }^{8}$. De façon générale, pour les périodes qui nous intéressent ici, le portrait - défini comme étant une œuvre (littéraire ou plastique) représentant une personne réelle ou fictive d'un point de vue physique et psychologique - sert à identifier un individu et à le mettre en valeur, grâce à la précision du vocabulaire descriptif ou par l'exactitude du dessin et de la couleur utilisés'. L'art du portrait est, en effet, l'un des points de rencontre du dire et du voir, deux domaines que les Grecs et les Byzantins ne distinguaient pas. Dans la langue grecque et byzantine courante, on utilise le plus souvent le mot ypá $\varphi \varepsilon ı v$ pour désigner l'action de décrire, d'écrire, de tracer, de graver ou encore de peindre. De même, le terme iotopeīv signifie raconter en images ou en mots.

La description scripturale des caractéristiques physiques et psychologiques d'une personne,

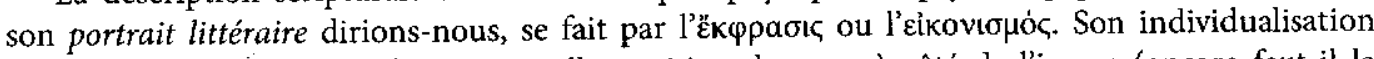
plastique, quant à elle, se fait soit par l'apposition du nom à côté de l'image (encore faut-il la nommer correctement), soit par la mise en valeur des attributs et des gestes les plus significatifs du sujet représenté, soit enfin par la mise en image, la plus fidèle possible, des traits physiques et

(4) Pour plus de détails, voir S. Lazaris, "Contribution à l'étude de l'hippiatrie grecque et de sa transmission à joccident (XIIle-XVe siècles)", dans M.-Cl. Amouretti, F. Sigaut (s.d.), Traditions agronomiques européennes. Élaboration et transmission depuis l'Antiquuité. Actes du 120' congrès national des sociétés historiques et scientifiques. Section Histoire des sciences, Aix-en-Provence, 23-25 octobre 1995, Paris, 1998, p. 143-149 et 155-156.

(5) Sur la période d'activité de cet hippiatre, voir S. LAZARIS, "Essor de la production littéraire hippiatrique et développement de la cavalerie: contribution à l'histoire du cheval dans l'Antiquité tardiven, dans M.-Th. Cam (s.d.), Actes du colloque international sur la médecine vétérinaire dans l'Antiquité (Brest, 9-11 sept. 2004) [Histoire], Rennes, 2007, p. 87-108. (http://halshs.archives-ouvertes.fr/docs/00/25/96/06/PDF/S.L.Medecine_veterinaire.pdh).

(6) G. BJöRCK, «Le Parisinus grec 2244 et l'art vétérinaire grec», $R E G, 48$ (1935), p. 511. Le philologue suédois s'est inspiré du litre donné dans un autre manuscrit grec, le Vaticanus Palatinus graecus 365 (f. 204r), un des manuscrits qui

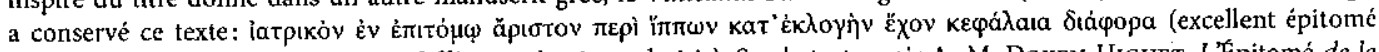
médical sur les chevaux, contenant différents chapitres choisis). Sur le texte, voir A--M. DOYEN-HigueT, L'Épitomé de la Collection d'hippiatrie grecque. Histoire du texte, édition critique, traduction et notes [Publications de l'Institut Orientaliste de Louvain, 54], Louvain-la-Neuve, 2006. Sur son illustration, voir S. LAZARIs, "L'illustration des traités hippiatriques byzantins: le De curandis equorum morbis d'Hiéroclès et l'Epitomè ", Medicina nei secoli. Rivista di storia della medicina, 11, 3 (1999), p. 521-546; lDEM, « Les rapports entre l'illustration et le texte de l'Epitomé, manuel byzantin d'hippiatrie», Archives internationales d'histoire des sciences, 143 (vol. 49 / 1999), p. 281-301.

(7) L'œuyre recomposée de Hiéroclès se présente en deux livres, chacun commençant par un prologue. Notre portrait ouvre le second livre.

(8) L. BRAUn, Iconographie et Philosophie, t. 2. Commentaires et Bibliographies [Cahiers du Séminaire de Philosophie, 13], Strasbourg, 1996, p. 57.

(9) Sur la notion d'individu et sur sa représentation au Moyen Âge, voir A. J. Gourevitch, La naissance de lindividu dans l'Europe médiévale, Paris, 1997; H. Belting, Pour une anthropologie des images [Le Temps des images], Paris, 2004, notamment chap. IV. Blason et portrait. Deux médiums du corps, p. 153-181; T. ToDorov, "La représentation de l'individu en peinture", dans B. Foccroulle, R. Legros, T. Todorov (s. la d. de), La naissance de l'individu dans l'art [Nouveau collège de philosophie], Paris, 2005, p. 13-39; Br. M. Bedos-Rezak, D. Iogna-Prat (s. d.), L'individu au Moyen Áge. Individuation et individualisation avant la modernité, Paris, 2005. 
psychologiques grâce au dessin d'après nature ${ }^{10}$. Description littéraire d'une part et, d'autre part, inscription nominative, attributs et gestes caractéristiques, traits reflétant l'apparence physique, constituent alors les différentes manières - associées ou non - pour composer le portrait d'une personne.

Le portrait reproduit dans l'œuvre recomposée de Hiéroclès ne peut, quant à lui, qu'être identifié par le biais des seuls attributs et gestes. Aucune inscription n'accompagne la représentation dans les deux manuscrits hippiatriques et les traits physiques ne peuvent pas être pris en compte puisque nous n'avons par ailleurs aucune description ou autre représentation de ce personnage. Cependant, avant d'analyser la gestuelle et les attributs de ces deux miniatures, quelques éclaircissements doivent être apportés sur l'art du portrait à Byzance et, plus particulièrement, sur celui de l'auteur.

\section{LE PORTRAIT À BYZANCE}

"Aujourd'hui encore, écrivait G. Millet, les murs blancs du "saloni" hospitalier sont parsemés de photographies. Les plus pauvres en désirent. Tout archéologue en voyage voit se dresser devant son objectif des silhouettes rigides et hiératiques pareilles aux Saints des icônes. Le papier albuminé inspire le même respect qu'autrefois l'encaustique ou la détrempe et reçoit les mêmes baisers. ${ }^{11}$ La commémoration des ancêtres par l'intermédiaire de leur portrait est une tradition ancienne. Au VIIle siècle, saint Damascène rapporte que «beaucoup dans leurs maisons ont peint des images d'hommes, les parents celles de leurs enfants ou les enfants celles de leurs parents, par affection mutuelle et pour ne pas les oublier ${ }^{12}$. Le témoignage de Damascène souligne cette relation si intime que les Byzantins ont développée avec les portraits, et avec les images de façon plus générale. Ils ont par là perpétué la tradition hellénistique du portrait et, contrairement à ce qu'on a pu soutenir ici ou $\mathrm{là}^{13}$, ils ont su différencier - hormis le portrait de l'empereur qui constitue à lui seul un cas à part ${ }^{14}$ le portrait des personnes civiles de celui des personnages saints ${ }^{15}$. Pour les premiers, d'ordinaire, les traits sont rendus grâce à une iconographie pénétrée d'un certain réalisme.

Ce type de portrait, le portrait privé, diffère donc souvent des autres, et ce tout au long de la civilisation byzantine. G. Millet l'avait déjà remarqué pour la production artistique des Paléologues, mais l'observation vaut pour toute l'histoire de l'empire byzantin. Il est, en effet, possible de détecter dans quelques œuvres, dès les premiers siècles de Byzance, les différences entre « the representations

(10) C'est d’habitude ce dernier procédé que nous associons le plus spontanément à la notion de portrait. Néanmoins, même si les deux autres procédés nous sont moins familiers, ce qui en résulte doit ètre considéré comme un portrait, du moins pour les sociétés et les périodes étudiées ici. Sur la définition du portrait médiéval, voir également l'introduction de J. Wirth dans A. Paravicini Bagliani, J.-M. Spieser, J. Wirth (textes réunis par), Le portrait. La représentation de l'individu [Micrologus' Library, 17], Florence, 2007, p. 9-15.

(11) G. Miller, "Portraits byzantins ", Revue de l'art chrétien, LXI (1911), p. 445-451.

(12) Damascène, Adversus Constantinum Caballinum, 2 (= $P G$, t. 95, col. 313).

(13) W. de GRüNEISEN, Le portrait, traditions hellénistiques et influences orientales, Rome, 1911, p. 97-99, critique sévèrement la production des portraitistes médiévaux, qui, selon lui, se seraient contentés d'appliquer des formules toujours plus simplifiées. Or, tous les types de portrait ne se prêtent pas à pareille critique.

(14) Un particulier (í̋tarıkóc) qui devient empereur entre dans un rôle. Désormais, il n'est plus seulement un homme investi d'une fonction, qu'il suffirait de représenter avec ses attributs. Il devient un homme qui prête momentanément son visage à l'Empire et qui est, en retour, définitivement transfiguré par cette fonction. Peu importe donc que son portrait ressemble à sa physionomie réelle: ce qui prime, c'est la fonction, l'empereur représenté doit ressembler à un empereur.

(15) Je fais allusion ici aux figurations qui n’évoquent aucune scène, aucun événement historique, mais simplement le Christ, les Saints ou encore la Vierge. 
of Saints and "true" portraiture ${ }^{16}$. Quoique ces deux types de représentations soient principalement le prolongement d'un faisceau de traditions liées à l'art du portrait antique, les chemins suivis par leurs concepteurs ne sont pas tout à fait les mêmes ${ }^{17}$. Aussi, tandis que l'on observe des efforts pour figurer les personnes civiles, remarque-t-on un net rejet d'une représentation fidèle à la nature quand il s'agit des personnages sacrés.

Ce refus d'une représentation réaliste commence très tôt, à en croire quelques attestations littéraires portant sur des œuvres anciennes disparues. Parmi les premiers témoignages connus, on citera les Actes apocryphes de saint Jean (26-29), textes composés entre 150 et 200 de notre ère ${ }^{18}$. Le passage commence par un récit des circonstances dans lesquelles un portrait de Jean fut exécuté à la demande de son disciple Lycomède, qui, dans sa chambre, rendit à l'image un véritable culte. Suit entre le mâtre et le disciple un débat sur la valeur et le sens de ce portrait que Jean conclut par ces mots : «Par la vie du Seigneur Jésus-Christ, ce portrait me ressemble. En fait, non pas à moi, mon enfant, mais à mon image charnelle. ${ }^{19}$. Dans ce texte - important pour comprendre les relations des premiers chrétiens avec les images - Jean désapprouve le culte rendu à son portrait et condamne la confection d'images réalistes, faites d'après nature. Ce type de portrait est aux antipodes du portrait iconique, qui ne représente pas l'image charnelle et visible d'un ètre mais va au-delà de l'art descriptif gréco-romain ${ }^{20}$. Dès lors, il ne s'agit plus de reproduire un personnage précis, mais ce qu'il incarne. D'ailleurs, Jean continue sa critique et reproche à Lycomède d'avoir fait quelque chose «de puéril et d'imparfait», car l'image peinte est "le portrait d'un mort» puisqu'elle ne représente que ce qui restera sur terre et non pas ce qui partira dans les cieux (Actes apocryphes de saint Jean, 28) ${ }^{21}$. Pour l'apôtre, le vrai portrait doit être celui de l'âme et non du corps fait d'après nature. Ainsi que le soutenait à juste titre G. Dagron, l'observation de visu est problématique dans l'art chrétien. On préfère imaginer que le peintre «travaille à partir d'une description orale (un eikonismos), ou que le saint lui apparaît en rêve ou vision, sous son identité véritable ou sous son nom d'emprunt. $»^{22}$.

La représentation des personnes civiles est en général très différente. Le portraitiste byzantin essaie de reproduire quelques caractéristiques physiques et psychologiques du modèle. Même si l'individualisation n'est pas des plus réussies, ce qui compte ce sont les efforts pour représenter les

(16) E. KITZINGER, «Some Reflections on portraiture in Byzantine Art», dans Mélanges Georges Ostrogorsky (=Zbornik Radova Vizantoloskog Instituta, VIII, [ [1963]), p. 187 (E. Kitzinger cite plusieurs exemples dans les pages qui suivent).

(17) Sur le sujet, voir A. Grabar, «Le portrait en jconographie paléochrétienne», dans M. Nédoncelle, B. Botte, L. de Bruyne, et al., Archéologie paléochrétienne et culte chrétien. Actes du colloque tenu à la Faculté [de l'Université de Strasbourg] du 7 au 9 décembre 1961 (= Revue des sciences religieuses, 36,3-4 [1962]), p. 87-109; S. SANDE, "The Icon and its Origin in Graeco-Roman Portraiture», dans L. Rydén, J. O. Rosenquist (eds.), Aspects of Late Antiquity and Early Byzantium. Papers read at a Colloquium Held at the Swedish Research Institute in Instanbul, 31 May - 5 June 1992, Uppsala, 1993, p. 75-84; A. GRABAR, Les voies de la création en iconographie chrétienne. Antiquité et Moyen Àge [Champs], Paris, 1994, notamment, p. 111-154 (réimpression de l'éd. de 1979); J.-M. SpIEser, "Invention du portrait du Christ», dans A. Paravicini Bagliani, J.-M. Spieser, J. Wirth (textes réunis par), Le portrait, op. cit., p. 57-76; G. DAGRoN, Décrire et peindre. Essai sur le portrait iconique [Bibliothéque illustrée des Histoires], Paris, 2008.

(18) W. Michaelis (übersetzt und erläutert von), Die apokryphen Schriften zum Neuen Testament [Sammltmg Dieterich, 129], Brême, 1956, p. 228-229 et 236-239.

(19) E. Junod, J.-D. Kaestul (cura), Acta Iohannis [Corpus Christianorum. Series Apocryphum, 1-2], Turnhout, 1983, t. 1, p. 177-181 el commentaire t. 2, p. 446-456.

(20) Sur le sujet, voir S. LAZARIS, "Au-delà de l'art: l'image cultuelle à Byzance», dans G. Siebert (éd.), L'expressio» du mouvement dans les iconographies de l'Antiquité, Actes du III colloque du Groupe Interdisciplinaire des Recherches Iconographiques de l'UMR 7044, Strasbourg 2-3 déc. 2005 (= Ktèma 32, 2007), p. 169-180.

(http://halshs.archives-ouvertes.fr/docs/00/25/95/97/PDF/S.L._ArtByzance.pdf).

(21) E. Junod, J.-D. Kaestli (cura), Acta Iohannis [Corpus Christianorum. Series Apocryphum, 1-2], Turnhout, 1983. t. 1, p. 178 et commentaire t. 2, p. 446-456.

(22) G. DaGron, «Mots, images, icònes", Destins de l'image (Nouvelle revue de psychanalyse), 44 (1991), p. 167. 
traits particuliers du visage, rarement déployés quand il s'agit des personnages saints. D'autres fois, bien entendu, ce n'est pas tellement l'individu qui est mis en image, mais son pouvoir et ses rapports avec Dieu ou avec ses sujets. Le portrait officiel, en l'occurrence celui de l'empereur, constitue un chapitre particulier et bien étudié de l'art du portrait byzantin sur lequel nous n'insisterons pas ici $^{23}$. C'est plutôt le portrait professionnel qui nous occupera. Quelques traits propres de la personne représentée y sont parfois rendus, mais c'est avant tout la catégorie socio-professionnelle du sujet figuré qui est mise en avant au moyen d'attributs significatifs.

Collectif (personnages en demi-cercle à l'intérieur d'une exèdre ou abside: on représente ainsi une assemblée de sages, un médecin en consultation ou encore le professeur entouré de ses disciples) ${ }^{24}$ ou individuel (un peintre en train de recopier une image ou un auteur devant son cuvre $)^{25}$, ce type de portrait est très développé au Moyen Âge. Parmi les représentations du portrait professionnel, la plus répandue est celle de l'auteur d'un ouvrage. En frontispice, il est reproduit dans les manuscrits médiévaux, le plus fréquemment au travail, en train d'écrire soit sur un rouleau, soit sur un codex, voire de copier de l'ún sur l'autre, de dicter son œuvre à un copiste, ou de la présenter au lecteur. Les miniatures médiévales accordent une part importante aux portraits d'auteurs. Parmi les plus anciennes représentations, mentionnons celles des évangélistes rédigeant leur évangile (fig. 1, Pl. I). Cette composition obéit à une formule iconographique relativement stéréotypée du portrait d'auteur.

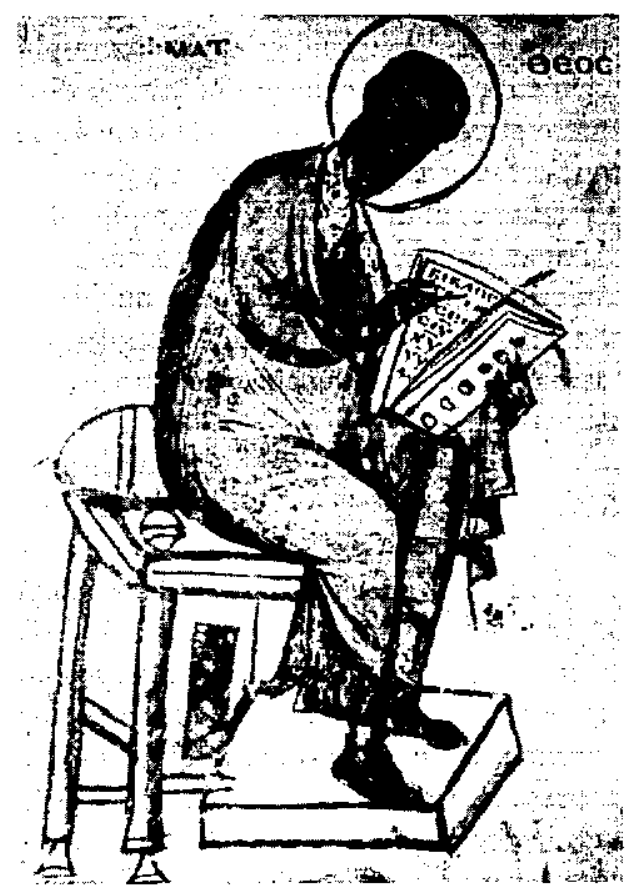

Fig. 1: Paris, Bibliothèque nationale de France, Suppl. 905, f. $54^{\mathrm{v}}$, VIII'-IX' ${ }^{\mathrm{c}}$ siècles (saint Matthieu) d'après Byzance et la France médiévale. Manuscrits à peintures du II $^{\mathrm{e}}$ au XVI $\mathrm{I}^{\mathrm{e}}$ siècle, Paris, $1958, \mathrm{n}^{\circ} 7$, p. 4 et pl. III)

(23) Sur ce type de portrait, voir A. GraBAR, L'empereur dans l'art Byzantin. Recherches sur l'art officiel de l'empire d'Orient [Publications de la Faculté des lettres de l'Université de Strasbourg, 75], Paris, 1936; 'T. Velmans, "Le portrail dans l'art des Paléologues », dans Art et société à Byzance sous les Paléologues. Actes du colloque organisé par l'Association internationale des Études Byzantines, Venise, sept. 1968 [Bibliothèque de l'Institut Hellénique d'Études Byzantines et Postbyzantines de Venise, 4], Venise, 1971, p. 91-148; I. Spatharakis, The Portrait in Byzantine Illuminated Manuscripts [Byzantina Neerlandica, 6], Leyde, 1976; C. HEAD, Imperial Byzantine Portraits. A verbal and graphic gallery, New Rochelle, NY, 1982; I. Spatharakis, "A note on the imperial portraits and the date of Par. gr. 510 ", JÖB, 39 (1989), p. 89-93; I. Kalavrezou, "Portraits and Portraiture», dans The Oxford Dictionary of Byzantium, t. 3, New York - Oxford, 1991, p. 1703-1704; G. DAGRoN, Décrire et peindre, op. cit..

(24) Voir quelques exemples dans le Dioscoride de Vienne (Wien, Österreichische Nationalbibliothek, medicus gr. 1, ff. $2^{v}$ et $3 v$ ); dans les Paris. gr. 36 (f. 187v) et 2243 (f. 10v) ou encore dans le Madrid, Biblioteca nacional, Vitr. 26-2, f. 134x. Reproductions dans O. MazAL (Kommentar von), Der Wiener Dioskurides. Codex medicus Graecus I der Österreichischen Nationalbibliothek [Glanzlichter der Buchkunst, 8, 1], Graz, 1998, ff. 2" et 3"; La médecine médiévale à travers les manuscrits de la Bibliothèque Nationale (Bibliothèque Nationale, 31 août-5 oct. 1982), Paris, 1982, $\mathrm{n}^{\circ} 21$ et commentaire p. 43; Byzance. L'art byzantin dans les collections publiques françaises (Musée du Louvre, 3 novembre 1992-1 février 1993), Paris, 1992, $\mathrm{n}^{\circ} 350$ et commentaire p. 454-455; V. Tsami KDA, The Illustrated Chronicle of Ioannes Skylitzes in Madrid, Leyde, 2002, fig. 321 et commentaire, p. 357-358.

(25) Voir quelques exemples dans le Paris. gr. 923 (f. 328v) et dans le Oxford, Bodleian Library, Barocci 87, f. 33*. Reproductions dans V.W. EgBert, The Medieval Artist at Work, Princeton, N.J., p. 24-25; I. SPatharakis, The Portrail in Byzantine Illuminated Manuscripts [Byzantina Neerlandica, 6], Leyde, 1976, fig. 182 et commentaire p. 258-259. 
Voici la description qu’en propose Brigitte Mondrain: «Selon le schéma régulièrement adopté, la représentation de l'évangéliste précède son cuvre: le saint est figuré assis, tourné de profil vers la droite, c'est-à-dire que, dessiné sur un verso de folio, il fait face à la page initiale de son Évangile, qui débute sur le folio suivant. Il tient sur les genoux ou, plutôt, sur le genou gauche un livre dans lequel il écrit les premiers mots de son texte, que le lecteur retrouvera aussitôt sur la page contiguë, tandis que, devant lui, se dresse un pupitre, monté sur un pied dont la base part d'un coffre. Le couvercle de ce meuble est au niveau du siège, dessus reposent en particulier l'encrier avec encre noire et encre rouge, une fiole d'encre noire et, parfois, un couteau destiné à aiguiser le calame ou, le cas échéant, à effectuer un grattage sur un folio du volume en cours d'élaboration, en cas de faute commise. ${ }^{26}$. L'iconographie du portrait d'auteur se confond alors avec celle du scribe. Tout au long du Moyen Âge, la grande majorité des images de ce type de portrait - aussi bien dans les auvres de contenu religieux que dans les textes profanes, y compris les traités scientifiques retient, justement, la formule iconographique de l'auteur-scribe. Or, cela n'est pas le cas dans les deux manuscrits hippiatriques illustrés de l'œuvre recomposée de Hiéroclès.

\section{LE PORTRAIT DANS L'GUVRE RECOMPOSÉE DE HIÉROCLẺS}

Dans les deux manuscrits, le personnage en question est assis sur un siège en bois, sans dossier mais pourvu d'un coussin. Ses pieds prennent appui sur un repose-pied, également en bois. Barbu, avec le visage hiératique du vieillard savant, il porte une robe et un manteau. Les deux représentations ne sont pas identiques. Dans le manuscrit de Leyde (fig. 2), l'homme est reproduit de trois-quarts face, la tête légèrement tournée vers sa gauche et, tel un personnage saint, il est auréolé. Il tient dans sa main droite un objet de forme cylindrique qui pourrait être un rouleau. Il lève l'autre main, paume tournée vers le haut, le pouce venant toucher le majeur et l'annulaire repliés. À sa gauche a été peint un objet difficilement identifiable (sorte d'armoire à livres, mal comprise par le miniaturiste?). Dans le manuscrit parisien (fig. 3), le personnage est également figuré de troisquarts, sauf la tête, qui est strictement de face, portant un regard aigu sur le lecteur du manuscrit. L'homme serre dans sa main gauche un long bâton ${ }^{27}$ et sa main droite, levée, est présentée de dos, les doigts fléchis dans un geste qui pourrait faire penser à celui du personnage du manuscrit de Leyde. Outre un pupitre vide, on a peint à l'arrière-plan à gauche un arbre schématisé qui est en grande partie caché

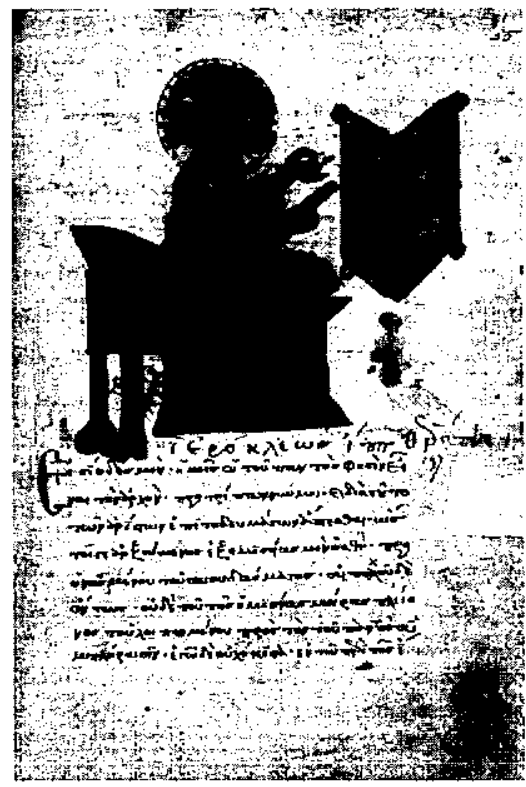

Fig. 2:Leiden, Bibliotheek der Rijksuniversiteit, Vossianus graecus Q. 50, f. 55r, milieu XIVe s. Leiden, Bibliotheek der Rijksuniversiteit(옹

(26) Br. Mondrain, Lire et écrire à Byzance [Centre de recherche d'Histoire et Civilisation de Byzance, Monographies, 19], Paris, 2006, p. 11.

(27) Dans les exemples que nous aborderons pendant l'analyse de cet attribut, le bâton est toujours maintenu dans la main droite. Il est toutefois difficile de savoir s'il est représenté ici comme gaucher ou s'il ne s'agit pas simplement de mettre en avant le geste de l'autre main. Sur l'iconographie des gauchers, en l'occurrence de l'auteur-évangeliste, dans l'art byzantin, voir I. Spatharakis, The Left-handed Evangelist. A contribution to Palaeologan Iconography, Londres, 1988. 
par un édifice architectural. Celui-ci est relié à une colonne, placée à droite de la composition, par une étoffe qui coiffe la scène. Elle est du même rouge que le toit du bâtiment. Même si la composition, dans les deux manuscrits hippiatriques, n'est pas la même, elle ne s'inscrit pas, ni pour l'un, ni pour l'autre, dans le type du portrait d'auteur-scribe. Aucun instrument d'écriture, aucun copiste ou livre ouvert (codex ou rouleau) - avec ou sans partie de texte - ne font, en effet, partie de cette scène. Dans l'un comme dans l'autre cas, la différence avec les portraits d'auteurscribe, tant dans l'art byzantin qu'occidental, est manifeste. Même constat quand on les compare avec les manuscrits hippiatriques occidentau $x^{28}$. Dès lors, de qui et de quoi s'agit-il ? Seule l'étude iconographique des attributs et des gestes pourrait aider à comprendre qui se cache derrière ces portraits et les intentions de leurs miniaturistes.

Le geste du personnage dans le manuscrit de Leyde (fig. 2) est peu attesté dans l'art byzantin et a souvent posé des problèmes d'interprétation. Myrtá Acheimastou-Potamianou, par exemple, hésite entre un geste de bénédiction et un geste de discours ${ }^{29}$. Toutefois, les gestes de bénédiction sont dirigés vers les fidèles et non, comme c'est le cas ici, vers le personnage lui-même. D'ailleurs, à en croire $M$. Didron dans ses commentaires sur le manuel d'iconographie chrétienne de Denys de Fourna, on ne doit pas, dans l'art byzantin, joindre trois doigts pour représenter une main qui bénit $^{30}$. De toute façon, dans le cas précis du manuscrit de Leyde, le fait que ce soit la main gauche qui fasse ce signe exclut le geste de bénédiction. On remarque ce signe le plus souvent dans le répertoire iconographique chrétien, notamment chez différents Saints et apôtres ${ }^{31}$. Quelques évangélistes, par exemple, sont ainsi représentés devant leur évangile, le calame dans une main et faisant de l'autre un signe identique à celui de notre personnage. Il s'agit alors très vraisemblablement d'un geste d'allocution et de transmission du savoir et de la bonne parole, lié à l'autorité de l'auteur. Ce caractère concret de l'autorité de l'auteur est même accentué dans ce cas par la représentation du début du texte de leur évangile. "Dans l'Antiquité et au Moyen Âge, écrit É. Palazzo, la notion d'auteur est au coeur de la culture chrétienne. Pour le christianisme de ces époques, l'auteur est celui qui possède "l'autorité" lui permettant d'écrire, de rédiger des textes valant pour tous les chrétiens. En effet, la notion d'autorité, l'auctoritas, est indissociable de la figure même de l'auteur $[\ldots] \aleph^{32}$.

Il est d'ailleurs fort probable que le miniaturiste du manuscrit de Leyde ait utilisé comme modèle une figure dérivant du répertoire iconographique chrétien. Le geste et, surtout, l'auréole dans le

(28) Voir par exemple dans les New York, Pierpont Morgan Library, MS. 735, f. 5; London, British Library, Add. 15097, f. $4^{r}$ (reproductions dans L. BRUNoRI CiANTI, L. CiANTI, La pratica della veterinaria nei codici medievali di mascalcia, Bologne, 1993, fig. 128 et commentaire, p. 285-297; fig. 134 et commentaire, p. 298-309).

(29) M. Acheimastou-Potamianou, Byzantine Wall-Paintings [Greek Art], Athènes, 1994, fig. 145 et commentaire p. 249, dans la description qu'elle donne de la représentation de l'apôtre Pierre à Saint-Georges de Staro Nagoricino, hésite entre un geste de bénédiction ou d'allocution ("gesture of blessing or speech»). Néanmoins, dans une représentation de saint Jean Prodrome, au geste identique, elle n'hésite pas à qualifier ce signe de geste de discours (cf. ibidem, fig. 71 et commentaire p. 229).

(30) A.N. DiDRon (introduction et notes par), Manuel d'iconographie chrétienne grecque et latine [Burt Franklin Research \& Source Works Series, 45], New York, N.Y., s.d., p. 455-456 (réimpression de l'édition de Paris, 1845).

(31) Voir quelques exemples dans G. Galavaris, The Illustrations of the Prefaces in Byzantine Gospels [Byzantina Vindobonensia, 11], Vienne, 1979, figs. 54 et 55 et dans Trésors du monastère Sainte-Catherine, Mont Sinaï, Égypte, Martigny, 2004, $\mathrm{n}^{\circ} 15$ et commentaire p. 90-92.

(32) É. PALAzzo, "Le portrait d'auteur dans les manuscrits du Moyen Âge», dans É. Palazzo, R. Rech, M. Bobin (textes de), Portraits d'écrivains: la représentation de l'auteur dans les manuscrits et les imprimés du Moyen Âge et de la première Renaissance [(Re)découvertes, 7], Poitiers, 2002, p. 21. 
manuscrit de Leyde renvoient effectivement à des représentations des évangélistes (fig. 3-4) ${ }^{33}$. Notons toutefois que la présence de l'auréole ici donne l'impression d'une copie aveugle transposée dans un autre domaine textuel, celui de l'hippiatrie. N'aurait-on alors pas pris soin d'adapter au contenu du manuscrit l'attribut principal de ce portrait? Peut-être. Toutefois, cette auréole pourrait ne pas être une erreur. Sa représentation s'expliquerait alors par la volonté du miniaturiste d'accentuer, avec cet attribut, l'autorité de ce personnage en le distinguant ainsi d'un écrivain ordinaire, lä̈que de surcroit, afin de donner plus d'importance à l'œuvre communiquée au lecteur du manuscrit. D'ailleurs, le fait qu'il ait choisi de le représenter non pas dans le type classique du portrait d'auteur-scribe confirmerait quelque peu l'idée qu'il aurait voulu différencier cette image en lui accordant ainsi une tout autre importance ${ }^{34}$. L'essentiel dans ce portrait n'est donc pas tant l'autorité de l'auteur du point de vue de sa profession mais plutôt de celui de l'éminence de l'œuvre transmise. Les raisons de ce choix, comme nous le verrons, sont très probablement les mêmes que pour le manuscrit parisien ${ }^{35}$.

Pourtant, bien que l'intention semble être la même pour les deux portraits, tout autre est le choix iconographique dans le Parisinus (ou déjà dans le modèle de ce dernier) ${ }^{36}$. Ici (Pl. II), l'autorité de l'auteur - dans la perspective de confirmer la sagesse de son ceuvre - est plutôt affirmée par sa représentation hiératique et de stricte frontalité ainsi que par le long bâton qui tient dans sa main. En communication directe avec le lecteur, cet angle de vue est réservé aux représentations dites "en majesté» et notamment à des personnages saints très

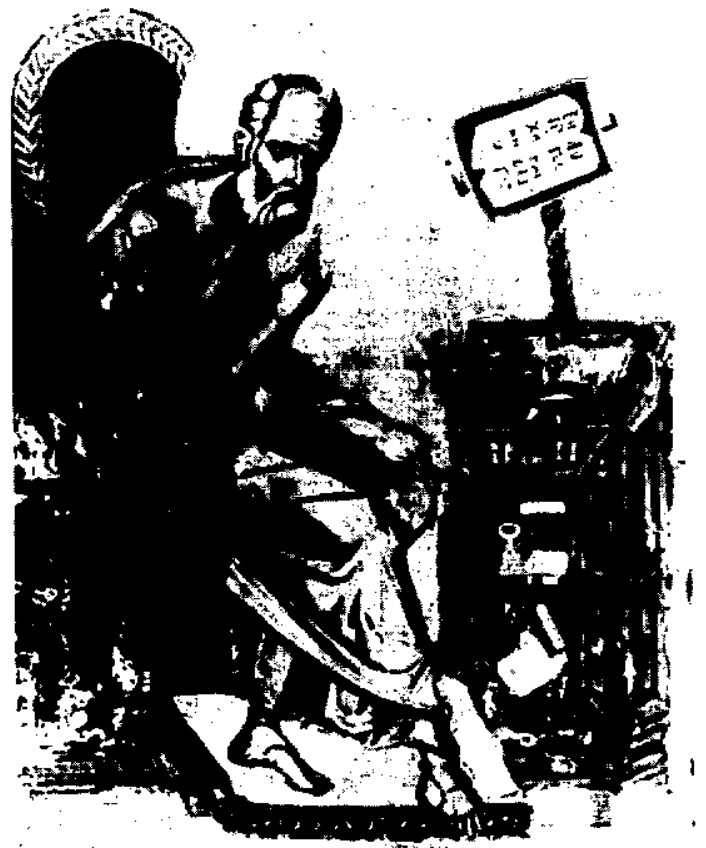

Fig. 3: Hagion Oros, Monè Vatopediou, 950, f. 158v, milieu du XIe siècle (saint Jean) d'après I. Spatharakis, The Lefthanded Evangelist. A contribution to Palaeologan Iconography, London, 1988, fig. 99

(33) Voir par exemple l'évangéliste Jean dans le Mont Athos, Vatopedi 950, f. $158^{\vee}$ (fig. 3) ou encore l'évangéliste Marc dans le Athina, Ethnikè Bibliothèkè tès Ellados, 57, f. 107v (fig. 4). Sur le premier, voir I. Spatharakis, The Left-handed Evangelist. A contribution to Palaeologan Iconography, Londres, 1988, fig. 99 et commentaire p. 63 et sur le second, voir R. S. Nelson, The Iconography of Preface and Miniature in the Byzantine Gospel Book [Monographs on Archaeology and the Fine Arts, 36], New York, N.Y.,1980, fig. 1.

(34) Je reviendrai sur le portrait du manuscrit de Leyde et les influences subies par son miniaturiste dans une étude à paraître prochainement.

(35) Ces deux manuscrits ne proviennent pas d'un modèle commun direct. Cependant, comme le soulignait G. Björck pour le texte, de même pour les images, il faut imaginer un archétype illustré autre que les modèles directs des deux manuscrits hippiatriques conservés (voir G. BJöRCK, "Le Parisinus grec 2244 », op. cit., p. 522).

(36) Plusieurs caractéristiques dans cette miniature trahissent, en effet, une copie provenant d'un modèle que je situc autour du XII siècle. L'imitation d'un modèle de cette époque n'est surprenante car l'écriture de ce manuscrit présente déjà des éléments archaïsants de cette mème période (voir S. Lazaris, « Le Parisinus graecus 2244 ", op. cit., p. 163-167). Parmi les miniatures de cette époque, citons l'apótre Matthieu dans le Oxford, Bodleian Library, Christ Church 12, f. 28v (f̂g. 8). Comme le signale G. Galavaris, "Christ the King": A miniature in a byzantine gospels and its significance ", $/ O ̈ B, 21$ (1972), p. 120, "The broad forehead of Matthew, for instance, the treatment of the hair and beard and the proportions of the figure would point to the end of the twelfth or the beginning of the thirteenth century". Ces mèmes caracteristiques se rencontrent également sur le visage du personnage du manuscrit de Paris. 
importants, tels le Christ ${ }^{37}$, mais aussi aux portraits de l'empereur et de certains personnages de la cour, comme également à quelques grands savants. Hippocrate (Pl. III) ${ }^{38}$ ou encore Constantin Manassès

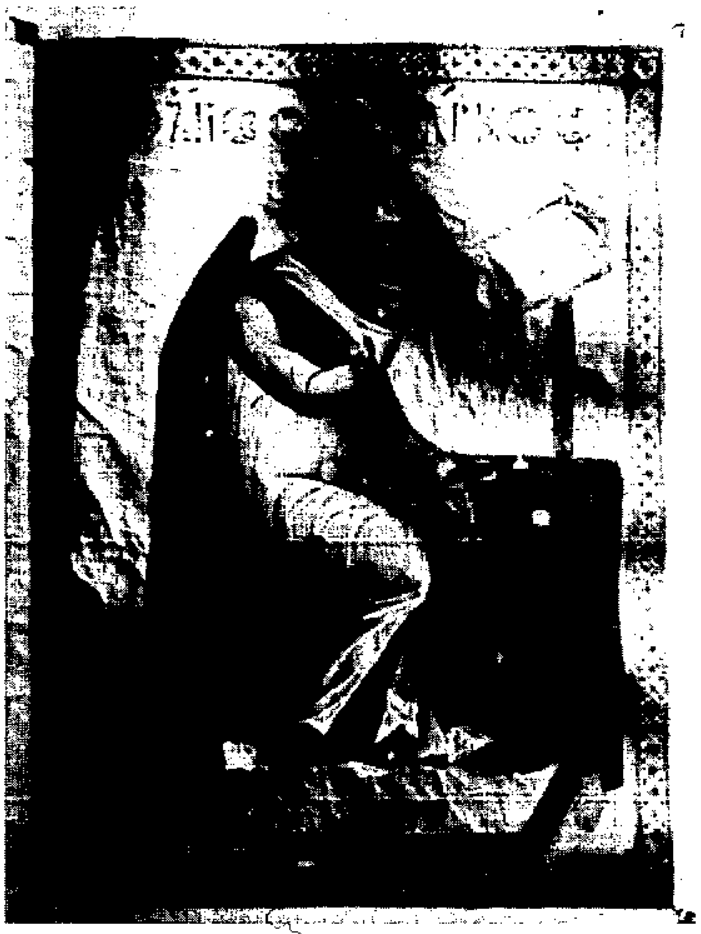

Fig. 4: Athènes, Ethnikè Bibliothèkè tès Ellados, 57, f. 107v, XII' siècle (saint Marc) d'après R.S. Nelson, The Iconography of Preface and Miniature in the Byzantine Gospel Book [Monographs on Archaeology and the Fine Arts, 36], New York, 1980, fig. 1.
$(\mathrm{Pl} . \mathrm{IV})^{39}$, entre autres, sont reproduits selon un angle de vue et une attitude hiératique semblables à ceux de l'homme du manuscrit hippiatrique de Paris. Notons cependant que, contrairement aux portraits de ces savants, le personnage du manuscrit de Paris ne fait pas allusion à son œuvre: aucun codex ou rouleau ouvert n'est reproduit; son regard s'impose de façon très soutenue et directe au lecteur du manuscrit ${ }^{40}$ et il ne tient aucun instrument d'écriture. Comme pour le portrait dans le manuscrit de Leyde, cette image n'est pas non plus un portrait d'auteur-scribe. Le personnage tient dans sa main gauche un long bâton et il fait, de l'autre main, un geste rappelant quelqu'un qui argumente devant un public ${ }^{4 !}$.

À première vue, le geste de la main gauche, mais aussi, dans une moindre mesure peutêtre, l'angle de vue avec le regard dirigé vers le lecteur et le long bâton, pourraient faire penser à un médecin enseignant son art. Toutefois, le petit nombre de représentations conservées de médecins les montrent d'ordinaire en consultation ou devant leur cuvre écrite. Quand ils sont figurés en train d'enseigner ${ }^{42}$,

(37) Parmi la pléthore d'images, voir dans le Paris, Musée du Louvre, département des Objets d'art, OA 2590. Reproduction dans Byzance. L'art byzantin dans les collections publiques françaises (Musée du Louvre, 3 novembre 1992Jer février 1993), Paris, 1992, n 162 et commentaire p. 252.

(38) Voir dans le Paris. gr. 2144, f. 10\%, approximativement daté de 1338 (le bifolium avec ces deux reprèsentations a été adjoint au reste du manuscrit dès sa réalisation et semble être de la même époque). Reproduction dans Byzance. L'art byzantin dans les collections publiques françaises (Musée du Louvre, 3 novembre 1992-1r février 1993), Paris, 1992, $\mathrm{n}^{\circ} 351$ et commentaire p. $455-458$.

(39) Voir dans le Wien, Österreichische Nationalbibliothek, hist. gr. 91, f. 1r, XIVé-XVIe siècles. La datation proposée est fondée sur les filigranes du manuscrit. En fait, il y a un filigrane de 1397, un de 1498, un de 1414-1422, un de 1504, un de 1490/92, un de 1472, un de 1500-11 et un de 1488 (cf. dans H. Hunger, Katalog der griechischen Handschriften der Österreichischen Nationalbibliothek, t. 1. Codices historici. Codices philosophici et philogici, Vienne, 1961, p. 94-102). Reproduction dans I. SpaTHARAKIS, The Portrait in Byzantine Illuminated Manuscripts, Leyde, 1976, fig. 101 et commentaire p. $159-160$ et 249 .

(40) L'auteur médiéval est le plus souvent représenté de trois-quarts et, quand il l'est de face, il regarde très rarement le lecteur-spectateur du manuscrit. À l'instar, par exemple, de Saint Basile dans le Milano, Biblioteca Ambrossiana, E. 49-50, f. 330', ses yeux, comme l'a déjà remarqué K. CoRRIGAN, Visual Polemics in the Ninth-Century Byzantine Psalters, Cambridge, 1992, p. 118, «are focused not on the viewer, but on his text». D'ailleurs, Hippocrate comme Constantin Manassès dans les exemples cités plus haut ne regardent pas de façon aussi directe le lecteur que le fait le personnage dans le manuscrit parisien.

(41) Plus précisément, il donne l'impression d'accompagner de cette main la scansion d'un discours, en décomptant les arguments sur ses doigts.

(42) Voir un exemple dans le Roma, Biblioteca Casanatense, 1382, f. $3^{\text {r }}$ (reproduction dans P.M. JoNEs, Medieval Medicine in Illuminated Manwscripts, Londres, 1998 (éd. revue), fig. 10). 
ils le sont comme tout enseignant, sans distinction particulière, en général assis sur un banc, l'index pointé vers un public présent ou imaginaire (fig. 5). Quelques exemples nous sont parvenus aussi bien dans le monde byzantin ${ }^{43}$ que dans le monde occidental ${ }^{44}$. La gestuelle ne correspond donc pas à celle du médecin-enseignant. Quant à son attribut principal, le bâton, il est depuis l'Antiquité l'insigne de celui qui détient l'autoritée ${ }^{45}$, en l'occurrence celle d'un homme savant. En effet, le personnage est ici figuré comme le sont les sages sur les mosaïques de Torre Annunziata (Musée de Naples) et d'Umbra-Sarcina (Musée de la Villa Albani à Rome $)^{46}$ ou encore sur une peinture murale dans la Catacombe de la Via Latina à Rome (fig. 6-7). Le geste et l'attribut se rapprochent donc plutôt ici du portrait d'un savant, d'un philosophe, qui transmet son art en argumentant devant un public qui n'est autre que le lecteur du manuscrit qu'il fixe, grâce à l'angle de vue choisi, intensément du regard.

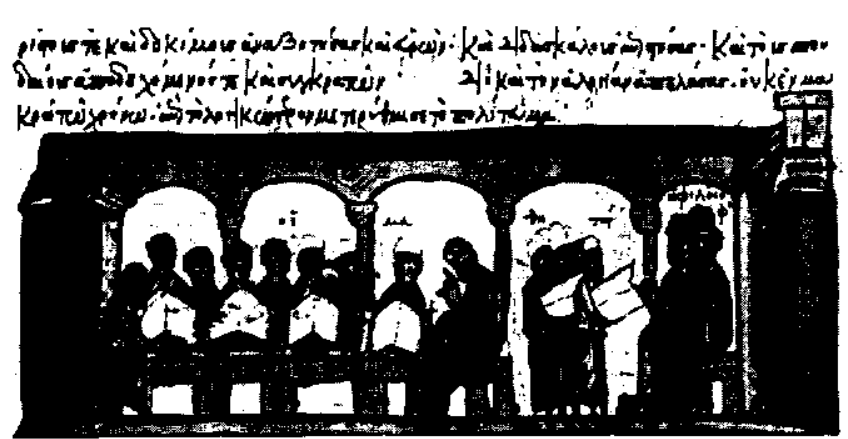

Fig. 5: Madrid, Biblioteca nacional, Vitr. 26-2, f. 134r (détail), XII ${ }^{e}$-XIVe siècles (enseignants et élèves) d'après V. Tsamakda, The Illustrated Chronicle of loannes Skylitzes in Madrid, Leyde, 2002, fig. 321

Rappelons-le, l'œuvre recomposée de Hiéroclès est divisée en deux livres, chacun introduit par un prologue. La lecture de ces deux prologues aide à mieux cerner la personnalité de l'auteur et, peut-être, à mieux comprendre les choix iconographiques dans les deux manuscrits hippiatriques. Dans ces prologues, il n'y a aucune allusion à la nouvelle religion de l'Empire. L'auteur se borne à des invocations, répétées, à la mythologie paienne et à des références littéraires provenant des œuvres anciennes. Hiéroclès évoque des hommes illustres de l'Antiquité, appartenant à des domaines variés: Euripide, Pindare, Asclépios, Simon d'Athènes, Xénophon, Aristophane de Byzance, Aristote, les frères Quintilii, Tarentinus, Hésiode, Micon l'Ancien. Hiéroclès, juriste de profession ${ }^{47}$ et très certainement amateur d'un passé révolu, pourrait finalement être représenté dans cette miniature non pas comme un quelconque auteur-scribe, mais comme un savant, dans la lignée des grands hommes du passé qu'il admirait. Construire ainsi son portrait, montre

(43) Voir dans le Madrid, Biblioteca nacional, Vitr. 26-2, f. $134^{r}$ et dans le Sinaï, Sainte-Catherine, gr. 4j8, f. 290r (reproductions dans V. TSAmaKDA, The Illustrated Chronicle of Ioannes Skylitzes in Madrid, Lcyde, 2002, fig. 321 et commentaire, p. 357-358; J. R. MARTIN, The Illustration of the Heavenly Ladder of Saint John Climacus [Studies in Manuscript Illumination, 5], Princeton, 1954, pl. LXXVII,216 et commentaire p. 187-189).

(44) Voir dans les Avranches, Bibliothèque municipale, 222, XIII siècle, f. $1^{\mathrm{r}}$; Dijon, Bibliothèque municipale, 525, XlVe siècle, f. $125^{\mathrm{v}}$; Paris, Bibliothèque Sainte-Geneviève, 1028, fin XIVe siècle f. $49^{\mathrm{v}}$. Le lecteur peut trouver des reproductions dans les bases de données "Enluminures», pour les deux premiers manuscrits, el "Liber Floridus", pour le dernier manuscrit. D'autres échantillons (par ex. le London, British Library, Harley 3140, f. 1' ou encore le London, British Library, Egerton, $737, \mathrm{f}^{\mathrm{r}}$ ) peuvent être consultés sur Internet, au Catalogue of Illuminated Manuscripfs de la British Library (http:/www.bl.uk/catalogues/illuminatedmanuscripts/welcome.htm). La base de données du Groupe d'Anthropologie historique de l'Occident médiéval (GAHOM) de l'EHESS m'a été également très utile.

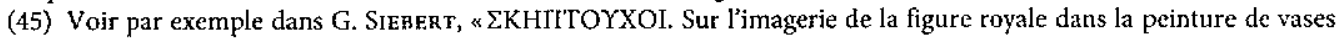
grecque", REA, 87 (1985), p. 263-288.

(46) Sur ces deux scènes, voir G.W. Elderkin, "Two Mosaics Representing the Seven Wise Men ", The American Journal of Archaeology, 39 (1935), p. 92-111 (reproduction, pl. XXII, a et b).

(47) Notons que sa baguette n'aurait pas pu faire office de bâton de justice puisque ce dernier est toujours tenu à la verticale et qu'il n'est pas utilisé pour désigner, comme le fait le personnage du manuscrit parisien. Voir quelques exemples dans R. JАСОВ, Images de Justice. Essai sur l'iconographie judiciaire du Moyen Âge à l'âge classigue, Paris, 1994, fig. 20, 23, 88 et 97. 


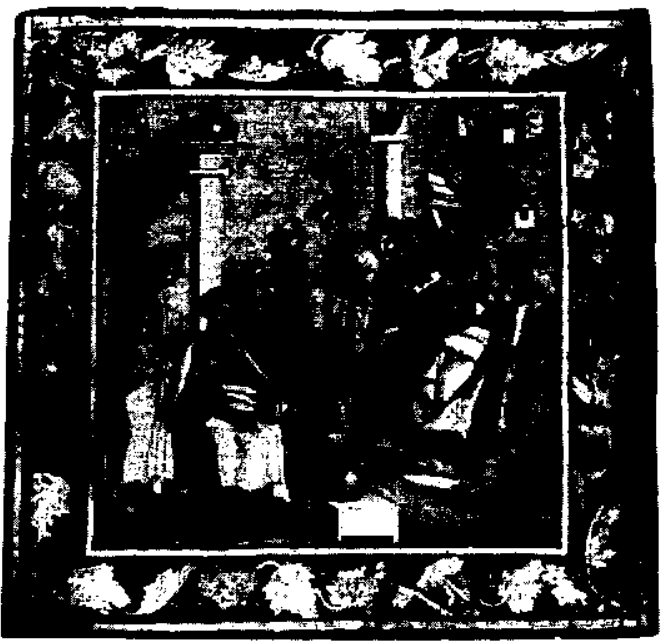

Fig. 6: Umbra-Sarcina, Rome, Villa Albani (assemblée de sages) d'après G. W. ElderkiN, “Two Mosaics Representing the Seven Wise Men ", The American Journal of Archaeology, 39 (1935), p. 92-111 (reproduction, pl. XXII, a et b.

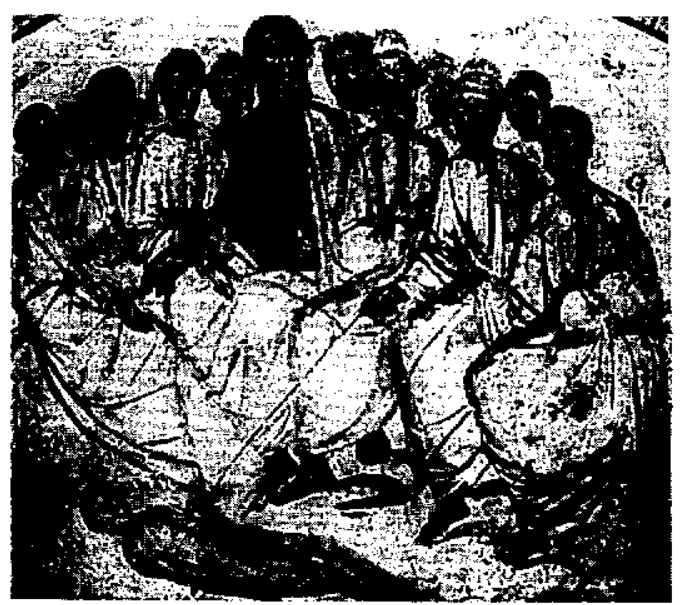

Fig. 7: Rome, Catacombe de la Via Latina, IVc siècle (assemblée de sages autour d'un corps étendu) d'après M. Grmek, D. Gourevitch, Les maladies dans l'art antique [Penser la médecine], Paris, 1998, fig, 143. une intention bien particulière: mettre en avant, plutôt que sa profession d'auteur, son autorité intellectuelle et, par conséquent, l'excellence de son ceuvre qu'il discute et transmet.

Les deux portraits paraissent donc atypiques face à l'habituel portrait d'auteur-scribe. Cependant, l'iconographie choisie, celle de l'auteur-savant, pourrait s'expliquer enfin par un détail important. Ce portrait n'ouvre que le second livre de l'œuvre recomposée de Hiéroclès et non, comme on aurait pu s'y attendre, le premier $^{48}$. Était-il prévu de réaliser un portrait d'auteur-scribe en frontispice du premier livre? Si l'on s'appuie sur le manuscrit de Leyde, le seul à avoir préservé le début du premier livre, aucun espace n'a été réservé sur le premier folio (actuellement f. $5^{r}$ ) pour une quelconque illustration. Toutefois, si le traité était précédé, dès l'origine, d'une table des matières ${ }^{49}$, on aurait pu imaginer qu'à la fin de celle-ci un portrait d'auteur-scribe ouvrît le traité qui suivait ${ }^{50}$. Dans ce cas, dans les deux manuscrits, la présence au début du second livre d'un portrait d'auteursavant s'expliquerait comme un pendant au portrait d'auteur-scribe du début du premier livre. Ce double portrait constituerait alors une double affirmation par l'image de l'autorité de l'auteur, véritablement considéré comme tel puisqu'on le voit, dans l'un, en train de rédiger son œuvre et, dans l'autre, en train d'argumenter et de la transmettre au lecteur du manuscrit.

En conclusion, l'étude de ces deux portraits montre que ce n'est pas tant l'individu, en l'occurrence Hiéroclès, qui est mis au premier plan, mais plutôt l'excellence de son œuvre qu'il nous lègue. Les deux miniaturistes représentent une idée plutôt qu'un personnage singulier. Les

(48) Soulignons toutefois que, dans le Parisinus, ce portrait se trouve actuellement au f. $\mathrm{l}^{\mathrm{r}}$, car, lors $\mathrm{d}^{\mathrm{t}} \mathrm{une}$ des restaurations qu'a subies ce manuscrit, on a cru, par erreur, qu'il s'agissait d'une miniature en frontispice de l'ceuvre. Dans le manuscrit de Leyde, il est silué au f. 55 r, à sa juste place.

(49) Celle qui occupe actuellement les folios $2^{\mathrm{v}-4^{\mathrm{v}}}$ dans ce manuscrit n'est pas de la même main que le reste du codex et elle a été, probablemenl, ajoutée par la suite (pour combler une perte?).

(50) La table des matières qui subsiste à présent est incomplète. D'après les calculs de A.-M. DoYEN-HiguET, L'Épitomé de la Collection d'hippiatrie grecque, op. cit., p. 120, «les quelque 120 titres restants occupaient peu ou prou trois pages, soit deux folios, qui seraient alors perdus après le fol. 4 ". Soulignons que dans ce cas, le premier cahier du codex ne pourrait pas être un binion mais plutôl un ternion, voire un quaternion, ce qui laisserait la place à une miniature. 
deux façons de la mettre en image témoignent des interrogations de chaque miniaturiste et de leur volonté respective de proposer une image conforme à la personnalité même de l'auteur grâce à une gestuelle et à des attributs savamment choisis ${ }^{51}$.

Stavros LAZARIS

UMR 7044, CNRS - Université de Strasbourg

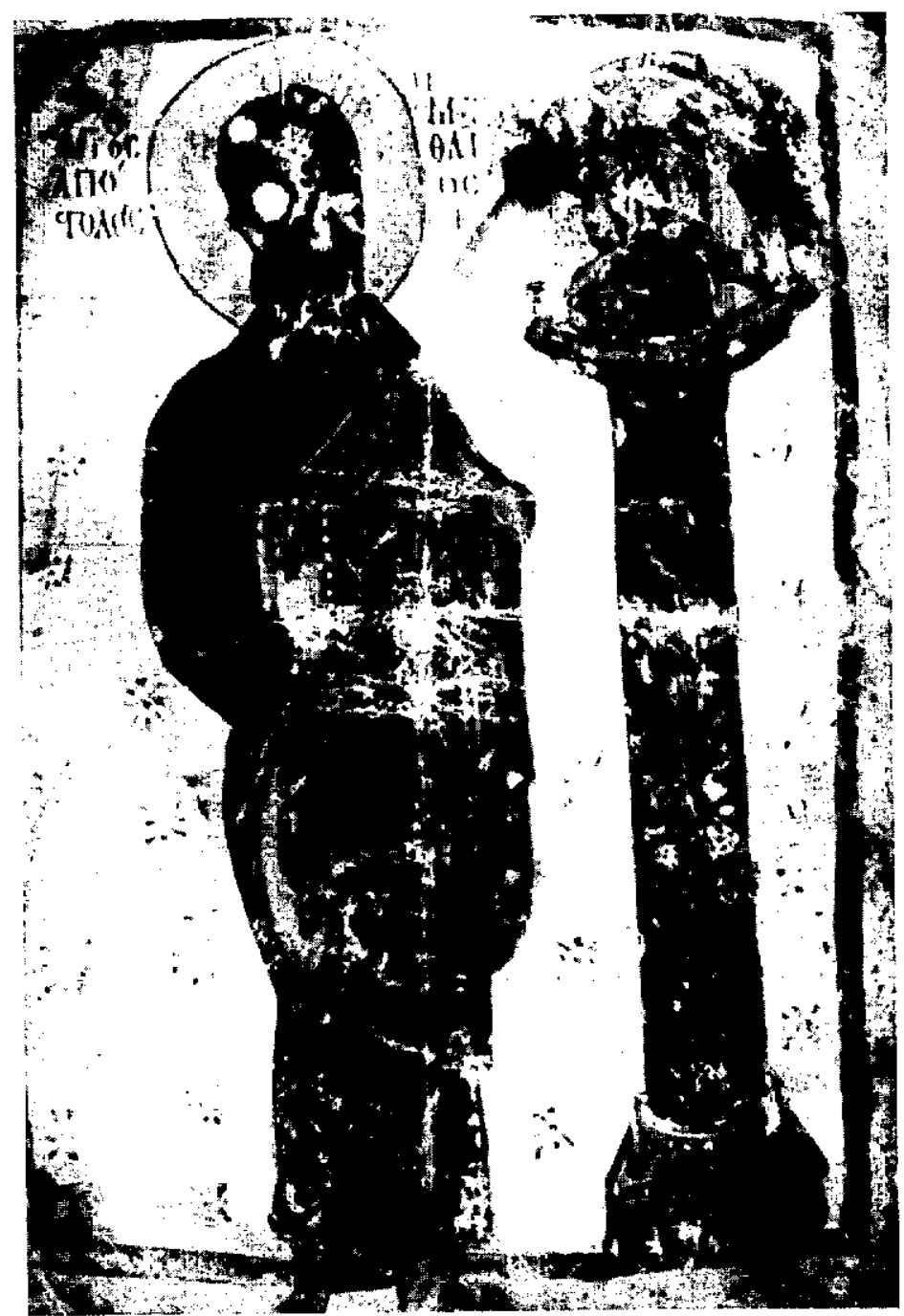

Fig. 8: Oxford, Bodleian Library, Christ Church 12, f. $28^{v}$, fin XIle-début XIII" siècle (saint Matthieu) d'après G. GalAVAR1s, "Christ the King": A miniature in a byzantine gospels and its significance " $J O ̈ B, 21$ (1972), fig. 1 .

(51) J'exprime mes plus vifs remerciements à MM. J. Durand et I. Spatharakis pour les conseils et les observations dont je leur suis redevabje dans la réalisation de ce travail. 


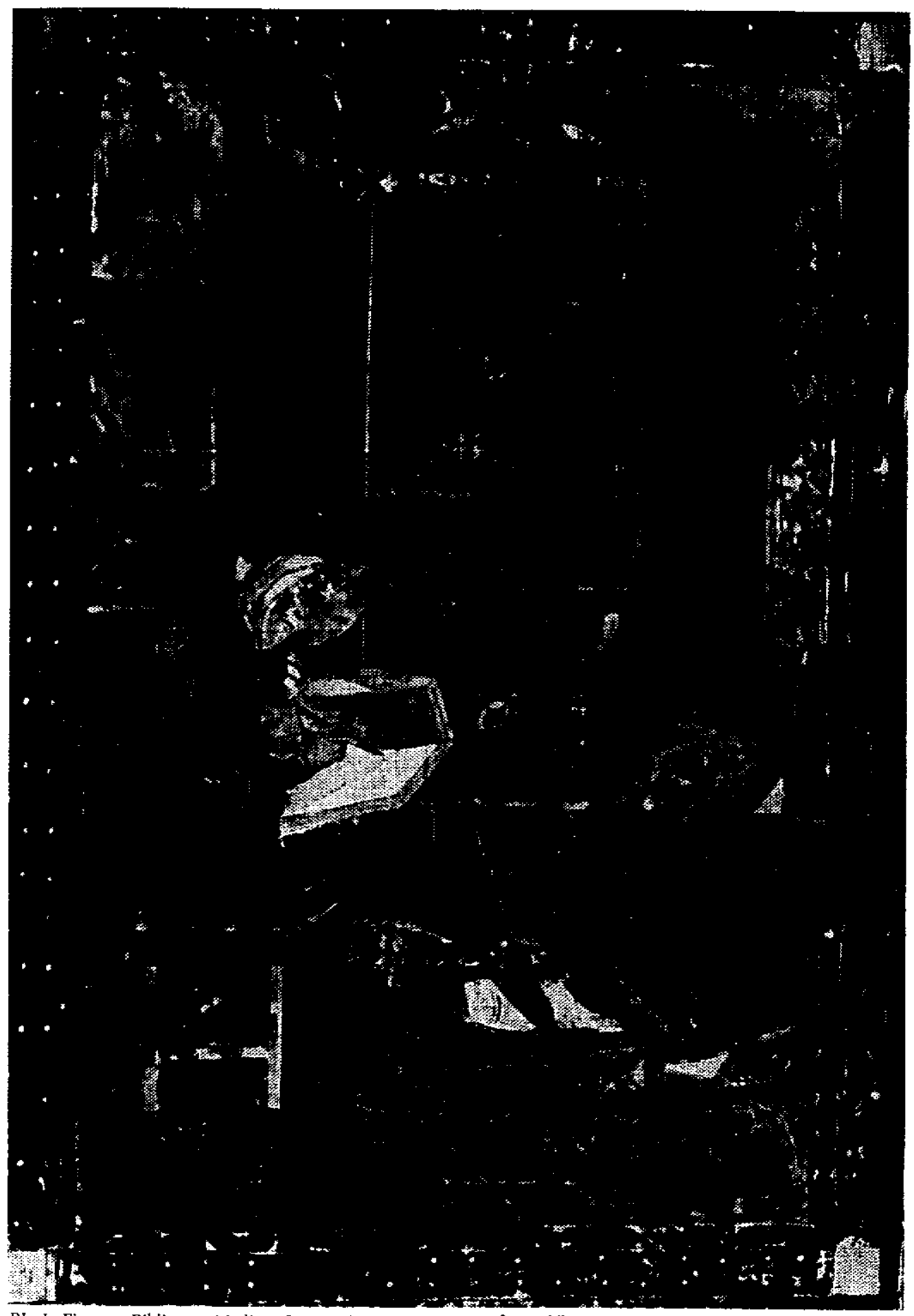

PL. I: Firenze, Biblioteca Medicea Laurenziana, Amiatinus I, f. 5a, début VIII e siècle (Esdras) d’après A. Grabar, C. Nordenfalk, Le haut Moyen Âge [Les grands siècles de la peinture], Genève, 1957, p. 119 
STAVROS LAZARIS

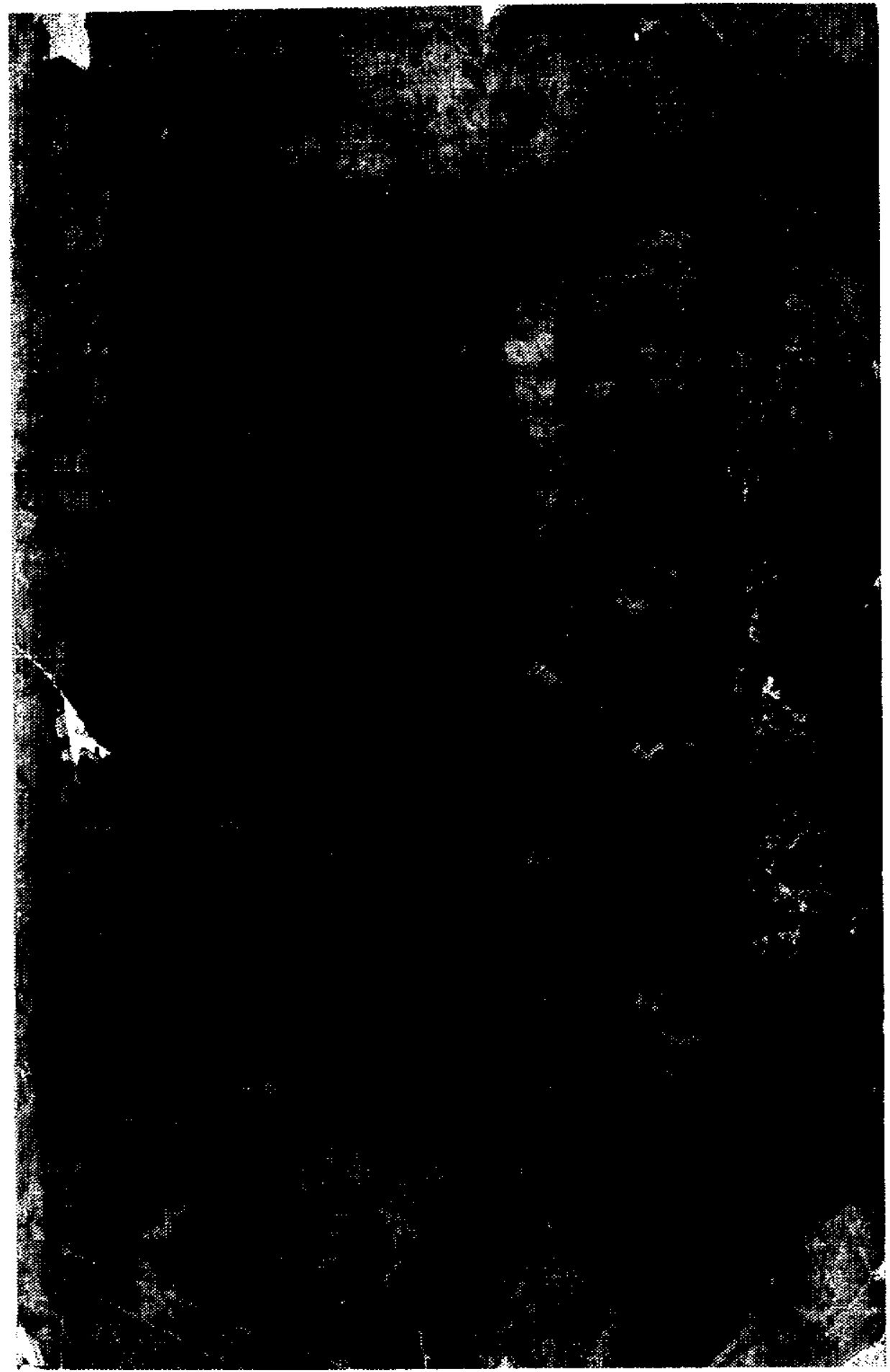

PL. II: Paris, Bibliothèque nationale de France, grec 2244, f, 1r, fin XIVe siècle Paris, @ Bibliothèque nationale de France. 


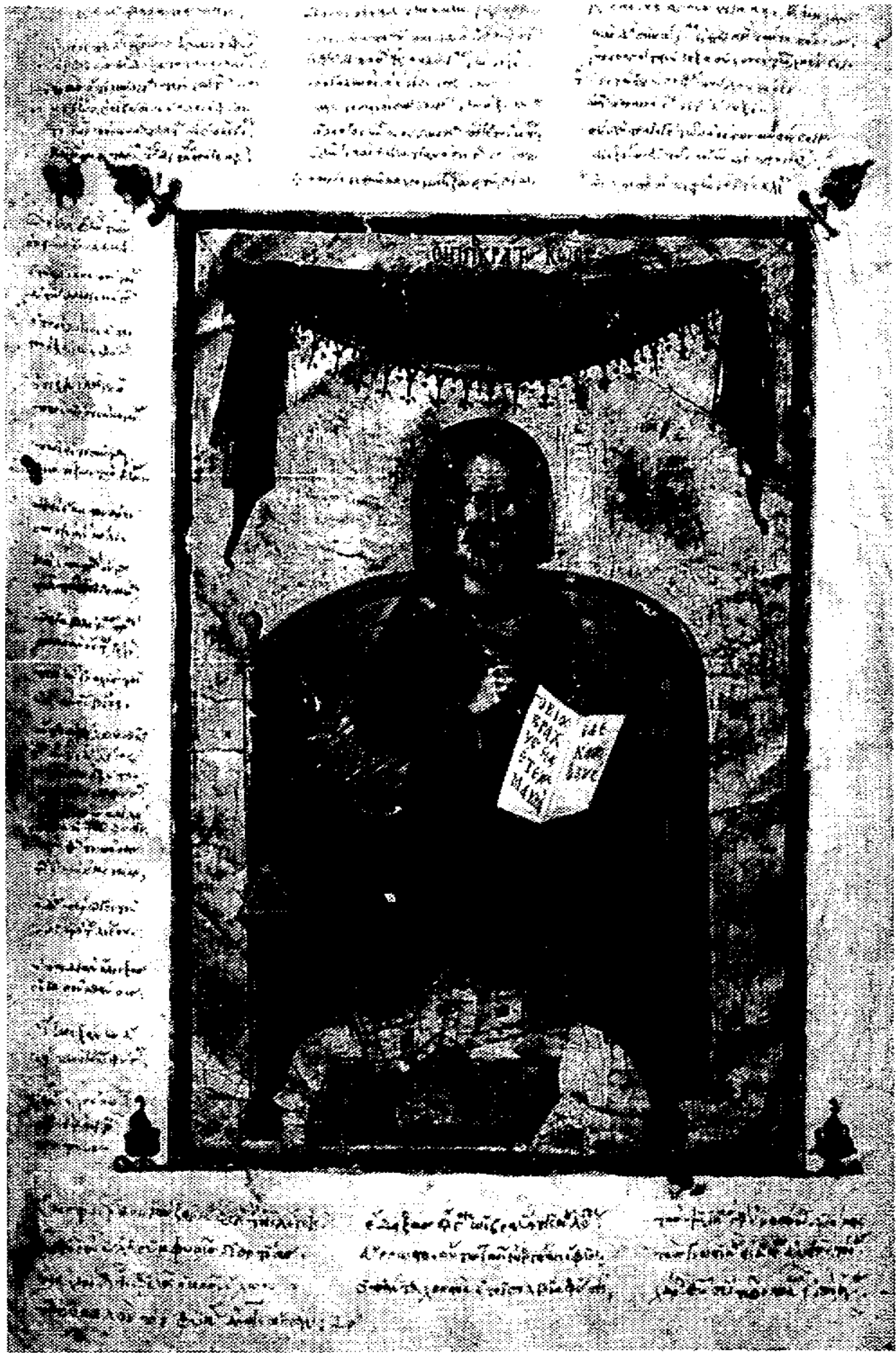

PL. III: Paris, Bibliothèque nationale de France, gr. 2144, f. 10, ca. 1338 (Hippocrate) d'aprés Byzance. L'art byzantin dans les collections publiques françaises (Musée du Louvre, 3 novembre 1992-ler février 1993), Paris, $1992, n^{\circ} 351$. 


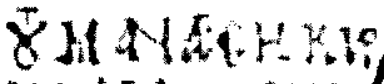

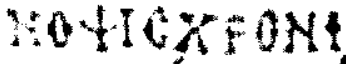

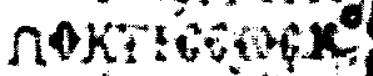

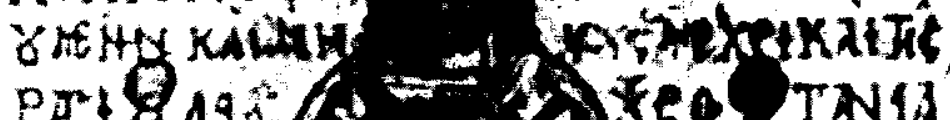

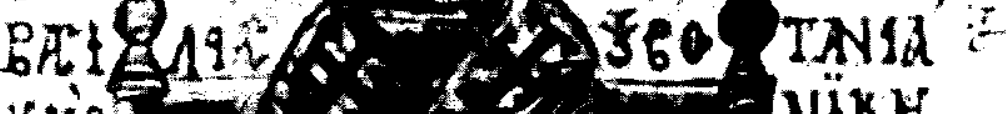

Kuir.

pos if

oim $6 e^{2}$
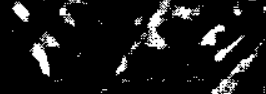

NikH

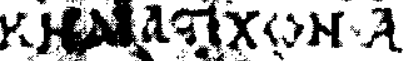

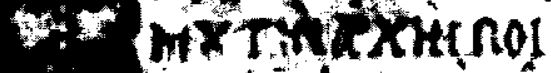

Riv $1+1$

TO

niv

f

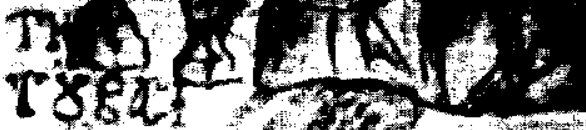

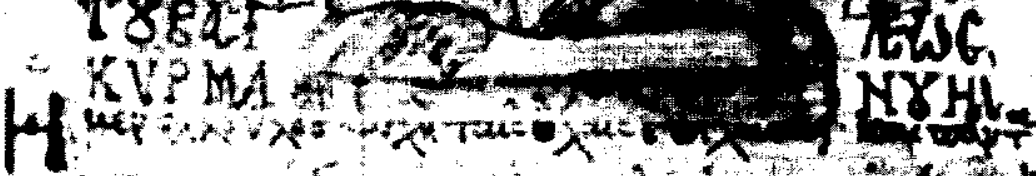

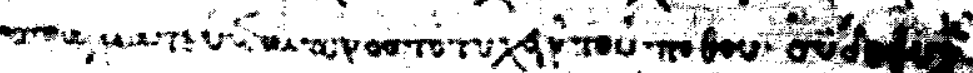

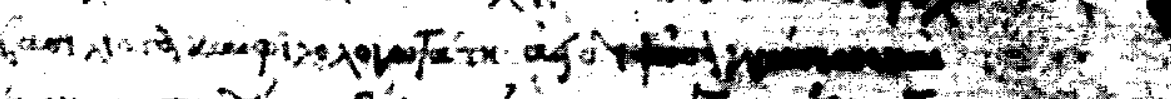

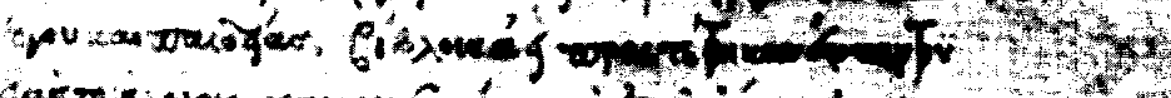

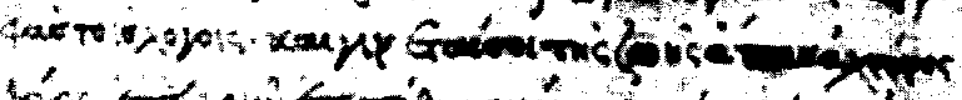

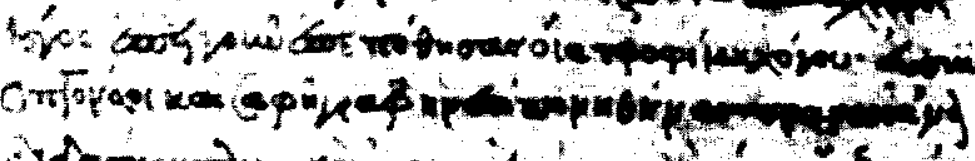

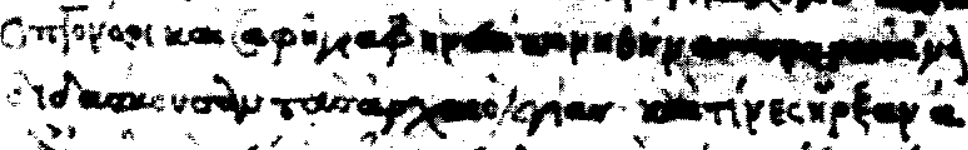

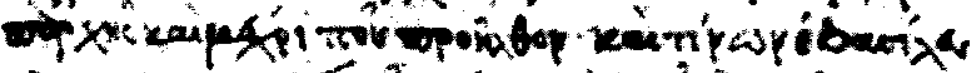

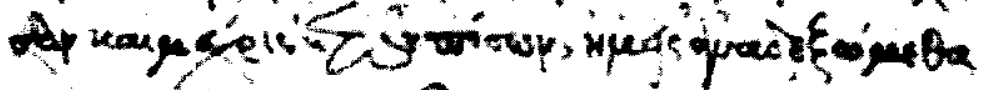

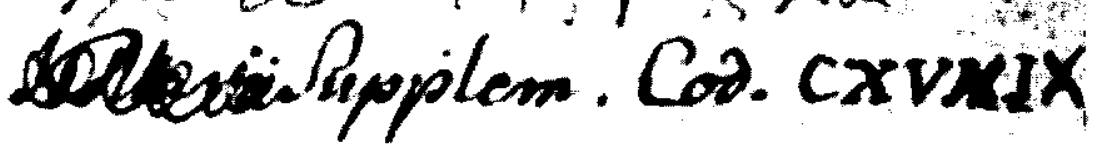

PL. IV: Vienne, Österreichische Nationałbibliothek, hist. gr. 91, f. 1; XIVe-XVie siècles (Constantin Manassès) d'après I. Spa'tharakis, The Portrait in Byzantine Illuminated Manuscripts, Leyde, 1976, fig. 101. 